\title{
COMMISSION 19: ROTATION OF THE EARTH (ROTATION DE LA TERRE).
}

President: Ya. S. Yatskiv

Vice-President: W. J. Klepczynski

Urganizing Committee: F. Barlier, H. Enslin, C. Kakuta, B. Kolaczek, I. I. Mueller, V.A. Naumov, P. Paquet, B. Proverbio, M. G. Rochester, Ye Shu-hua, K. Yokoyama.

\section{Introduction}

During the period, work on the problem of the Earth's rotation has continued to expand and increase its scope. The total number of institutions engaged in the determination of the Earth's rotation parameters (ERP) by different techniques has been increased significantly. The rotation of the Earth is currently measured by classical astrometry, Doppler and laser satellite tracking, laser ranging of the Moon, and radio interferometry. Several long time series of the ERP are available from most of these techniques, in particular, those made during the Main Campaign of the MERIT project. The various series have been intercompared and their stability, in the time frame of years to days, has been estimated for the purposes of establishing a new conventional terrestrial reference system (COTES). On the other hand, the difficulties of maintaining a regular operation for laser ranging to the Moon (LLR) have been recognized. It resulted in the proposal to organize an one-month campaign of observations in 1985 in order to complement the COTES collocation program and to allow additional intercomparisons with other techniques.

The international, electronic exchange of data on Earth rotation through the General Electric Mark III Information System computer network has been expanded during the period of this report. Many Time Service publications are exchanged in the RC28 catalog.

\section{Reference systems for study of the Earth's rotation}

Different aspects concerned with the definition and the establishment of reference coordinate systems for the Earth's dynamics, in particular COTES, have been considered by the IAG/IAU Working Group (Chairman I.I Mueller). It has been recommended that the reference frame of the future COTES be defined by an adopted set of spatial coordinates of a global network of observing stations.

C. Boucher and M. Feissel (1984) proposed a new realization of the BIH terrestrial system, including the, scale unit origin and orientation of the axes on the basis of the permanent stations used in the monitoring of the Earth's rotation by space geodesy techniques.

In the development of a celestial radio reference frame there are now over 100 sources whose relative positions are known with an average uncertainty less than 5 millarcseconds (J. L. F anselow et al, 33.043.003).

In the improvement of a celestial stellar reference frame there are now about 100000 stars, whose relative positions will be observed by the HIPPARCOS Space Astrometry Mission (P.L. Bernacca, 1983).

The orientation of the dynamic reference frame realized by the JPL ephemerides DE 200/LE 200 has been considered by E. M. Standish (32.047.016).

The effects of adopting new precession and equinox correction have been discussed in connection with a new definition of UT (31.044.001, 31/044.003, 31.043.010, 33.044.021).

J. Vondrak (31.045.001, 33.044.035, 33.044.061,34.044.004) derived the direct and indirect influences of the planets on nutation. The total effect is comparable with the 
smallest terms in the luni-solar nutation series adopted by the IAU. Some authors have considered the definition of the celestial ephemeris pole (32.043.010, 33.044.078).

The work on the PZT/DHI 82 star catalogue with improved star positions and proper motions has been completed at DHI, Hamburg. In addition, the improved catalogue PZT8 was completed at ZIPE, Pottsdam.

Yokoyama (1983, 1984) presented a full description of the method for reducing the optical astrometric observations at each station, as well as at the international centers, in accordance with the adoption of the FK5 system and the IAU (1976) System of Astronomical Constants for Jan. 1, 1984 onwards. This new method was presented to the 2nd MERIT workshop and was approved as the MERIT standards.

\section{Determination of the Earth's rotation parameters from the classical technique}

(Research in Instrumentation)

T. Tsubokawa has designed a new type of fully automatized pendulum astrolabe controlled from a remote minicomputer. It has been constructed and tested at Mizusawa (31.032.011, 33.032.036). The photoelectric astrolabe has been improved at CERGA-Grasse (33.032.009). Different origins of errors of observations by photoelectric astrolabes in the People's Republic of China have been studied (33.041.040, 33.041.050, 33.044.044). The photoelectric astrolabe of Yumman Observatory has proven to be the best in time observation of a dozen Chinese instruments. The relation between temperature and the displacements of the focal plane in the Danjon astrolabe of Wuchang Time Observatory has been derived (34.032.050).

At Irkutsk, a latitude service has been established using the observations from two Uanjon astrolabes (34.044.009). Regular observations of latitude and UT variations with the use of the Danjon astrolabe were started in Borowiec in 1982 (M. Lehmann).

Studies on the instrumental errors of the PZT are being continued at Tokyo (34.03.046) and at Kitab (34.032.022). Z. M. Malkin (34.032.062) has proposed an optimum construction of a pavilion for the PZT at Kitab.

Many papers concerned with the instrumental errors of photoelectric transit instruments and also with their automation have been published (34.032.011, 34.032.017, 34.032.041), 34.032.042, 34.032.045, 34.032.105, 34.036.106).

Research on instrumental errors of zenith-telescopes is being continued at Kitab (33.032.019-33.032.21). PZT observations at the DHI, Hamburg will be terminated by the end of 1986.

\footnotetext{
(Analysis of observationaldata and determination of the ERP)
}

N. I. Panchenko and A. A. Slavinskaya (34.044.024) investigated mean latitude variations of some stations equipped by astrolabes. Tidal and nutational effects from observations of the Paris astrolabe have been derived (31.043.012, 32.045.011). P. I. Popova has (34.044.022) determined the fortnightly nutation term from observations of bright zenith stars in Poltava. At Pulkovo, non-polar latitude variations derived from observation with two zenith telescopes (ZLL-180 and ZTF-135) have been studied (32.045.025, 32.045.026). New reduction and analysis of observational data by the $Z T L-180$ at Kitab have been undertaken (34.044.010-34.044.012).

Different systematic effects (wind influence, parallel curvature ets.) have been investigated at Poltawa (34.044.013, 34,044.023) and at Pulkovo (34.044.015). 
N. Capitane and N. Xiao (31.044.002) studied the Z-term of latitude and the w-term of $U T$, as computed by the $B I H$, for deriving the principal terms of nutation.

G. P. Pil'nik (32.044.040) estimated the power spectrum of UT determinations from 1968 to 1978 and showed that there were diurnal and semidiurnal variations.

R. M. Rasulov (34.044.011) determined corrections to the ILS pole coordinates resulting from micrometer errors for interval 1938.4-1939.2.

S. Takagi (32.031.503), A.A. Korsun' and R. Mi. Rasulov (34.044.021), D. Yu. Belotserkovskij and M.B. Kaufman (33.044.028) considered the determination of ERP in an uniform system, based on different sets of data. Re-reduction of observations of the Soviet Time Service from 1955 to 1979 has been done by L. I. Yagudin (33.044.036). J. Hefty (31.044.005) derived the Love number $K$ from UT determinations during the years 19671978.

Different aspects of Chinese activity in the field of ERP determination and analysis have been presented at the Shanghai Syposium on Astrometry (34.044.032-34.044.059) and have been published in Publs. Shanghai Astron. Obs. (33.044.078-33.044.094) and in Ann. Shanghai Obs. (33.044.065-33.044.070).

P. Fellget (34.044.005) computed the power spectrum of the observed variations of latitude, and calculated the explicit likelihood distribution.

V. L. Gorshkov (34.036.028, 34.036.059) proposed a modification to the traditional chain method in order to determine a correction for instrumental and refeaction errors.

S.-Y. Zu (33.044.029) and S. Okasaki and T. Sakai (34.044.094) considered the possibility of predicting polar motion and variation in the rate of the Earth's rotation respectively. Investigations of local, nonpolar variations in latitude determined by astrometric methods were carried out at the Space Research Center, Warsaw. Annual and semiannual terms of local latitude variations are very changable especially for some stations. Their amplitudes and phases have periodical variations with the periods of 2,3,4, years and longer. Introducing variable annual and semiannual terms of local variations of latitude instead of the annual mean as determined by the $\mathrm{BIH}$ ought to improve astrometric determinations of pole motion. About 20 astrometric stations have more stable local variations of latitude and UTO (B. Kolaczek and G. Teleki, 1982; B. Kolaczek, 1982; B. Kolaczek and B. Napiorkowska, 1983)

\section{Determination of the Earth's rotation parameters from the new techniques \\ (Laser)}

Instrumentation of the SLR stations was discussed at the Laser Ranging Workshop at Herstmonceux in September 1984. Different requirements of station distribution for the determination of ERP are discussed with the aim of minimizing the number of SLR stations 34.045.021) and the number of LLR stations (34.044.028).

D.E. Smith et al (34.044.105) derived the variations in the length-of-day from Lageos data.

The McDonald Observatory LLR data have been analysed for determining UTI and variations of latitude by $W$. Jin for the period 1971-1979 (32.044.043) and by J.O. Dickey and J. Q. Williams for the period 1972-1982. (34.044.029).

At present there are about $10 \mathrm{SLR}$ stations with an accuracy better than $10 \mathrm{~cm}$. One of them is at Simostato Hydrographic Observatory $(33.032 .037)$. 
D.C. Christodoulidis and D. E. Smith (34.045.023) discussed the role of SLR through the 1990's.

Two programs for analysing SLR data have been developed in the USSR. One used an analytical approach (Sternberg Astronomical Institute, Moscow) and the other used numerical integration (IVain Astronomical Observatory, Kiev.)

A permanent Doppler station (TRANET II) for satellite tracking is in operation since August, 1983 at the Observatorio de Marina de San Fernando, Spain. The station took part in the MiERIT campaign and the MEDOC campaign. Also, regular observations of Doppler satellites were made at Borowiec with the use of the Astro-receiver DOG-2, constructed at the Space Research Center.

(R adio interferometry)

The present state and future developments for the VLBI technique were discussed at an International Conference held in Toulouse in 1982 (33.012.020). The Mark III VLBI system allows recording and later processing of up to 112 megabits per second from each radio telescope of an interferometer array. The relative delays of the signals recived at each pair of sites can be determined with uncertainties under 50 psec (33.033.010). This information can be used for determination of the ERP (33.033.019) and

nutation motion (32.044.005). E.C. Pavlis and I. I Mueller (34.045.006) considered the effect of Earth's rotation errors in baseline determination.

Different aspects of the VLBI observations (frequency standard effect, method of observation and analysis etc.) and CERI have been considered (33.044.023, 34.036.234, 34.036.244, 34.044.081, M. В. Bondarenko and M. I. Yurkina, 1984).

\section{Intercomparison of different techniques for determining the Earth's rotation parameters}

Several papers concerned with the intercomparison of different techniques for determining the ERP have been presented at the IAU Coll. No, 63. "High-precision Earth rotation and Earth-Moon dynamics" (31.012.035). The colloquium was organized by

U. Calame, CERGA.

M. Feissel (31.044.059) considered the possiblity of determining ERP's independent of the observation technique.

ఈ. Kolaczek et al (1984) compared the pole position determined by different techniques and detected short periodical terms with periods in the following ranges of days: $70-80 ; 50-55 ; 30-35$, and $15-25$.

\section{Research concerned with the rotation of the Earth}

Several review papers concerned with dynamics of the Earth and its internal constitution (34.181.001, 34.081.014 - 34.081.020, 34.081.032, 34.044.103 - 34.044.104) have been published.

V. P. Dolgchev (34.044.089) obtained an explicit expression for the axial rotation of the rigid Earth and analysed secular and periodic components in the rate of the Earth's rotation.

M. Bur (32.044.041, 32.044.042) discussed the solution of the equations describing the rotation dynamics of a deformable Earth. He determined the deformation caused by the Earth's rotation.

J. M. Wahr (32.045.002, 34.044.001) examined the effects of the atmosphere and oceans on wobble excitation and variations in the length of day. He found that the 
atmosphere and ocean were apparently not the primary excitation source. R.T. Barnes et al (34.044.003) re-examined an angular momentum exchange between the atmosphere and solid Earth. Daily values of the atmospheric angular momentum functions have been calculated for period 1981-1982 and have been comapred with the ERP.

J. Hinderer et al (32.045.006) derived the basic equations describing the dynamical effects of the Earth's fluid core for an ellipsoidal Earth model without axial symmetry. This problem has been also studied by M. J. Sevilla and his collaborators at Instituto de Astronomia y Geodesia, Madrid. the Earth.

S. M. Molodenskij (1984) considered the theory of tide and nutation for real model of

S. K. Runcorn (32.044.006) considered the role of the core in irregular fluctuations of the Earth's rotation and the excitation of the Chandler wobble. The torque is supposed to be of an impulse character.

N. N. Parijskij (32.044.002) pointed out a correlation between irregular fluctuation and changes in gravity. However, new observations did not support this suggestion (Parijskij, 1985).

On the other hand, a comparative analysis of the irregularities in the Earth's rotation and the solar activity have been undertaken $(32.044 .003,32.044 .012)$.

C. F. Yoder et al (31.044.004) and J. B. Merriam (31.044.007) considered short period terms in the variation of the Earth's rotation. New determinations of secular and decade fluctuation in the Earth's rotation have been carried out by S. J. Goldstein using old solar tables (31.044.060) using timings of lunar eclipses and occultations. Besides the tidal deceleration of the Earth, the study, which is being continued (31.044.061, 33.012.006), found an acceleration component. Atmospheric excitation of the Earth's rotation has been discussed in details at the meetingof the IUGG/IAG Special Study Group 5.98 (Chairman J. 0 . Dickey). Abstracts for this meeting were printed in EOS, Transaction, AGU, 65, 16, 186, 1984.

The theoretical and observed estimates of the Chandler component in polar motion is being continued. B. Guinot (32.045.005) derived the amplitude, phase and ellipticity of this component based on data from 1900 to 1980. W.E. Carter (32.045.004, 33.004.005, 33.044.057) suggested that the Chandler component may be frequency modulated as a linear function of the polar motion magnitude.

C. Pan (32.044.026) explored the hypothesis on the multiple-frequency Chandlerian nutation. On the other hand, S. O. Kubo (32.045.016) did not find any positive evidence for the variable period hypothesis. He also discussed the damping factor $Q$, as derived from the Chandle wobble.

\section{Report of the Bureau International de l'Heure}

The BIH is organized in two sections: (a) Time, (b) Rotation of the Earth, respectively under the scientific responsibility of $B$. Guinot and $M$. Feissel.

This following report covers the activities on the Rotation of the Earth and related problems. The BIH activities on atomic time and some administrative and operational matters common to both sections are reported to Commission 31 .

\section{Current evaluation.}

The Earth Rotation Parameters (ERP), i.e., Universal Time and the coordinates of the pole, are evaluated regularly on the basis of independent time series obtained by all the existing techniques of observation: Very Long Base Radio-Interferometry (VLBI), 
Connected Element Radio-Interferometry (CERI), optical astrometry, Lunar Laser Ranging (LLR), laser ranging to LAGEOS (SLR), and Doppler tracking of artificial satellites.

The BIH has continued the computation of a solution by optical astrometry, from the observations of 88 stations in 22 countries. The IAU 1976 System and the IAU 1980 Theory of Nutation have been introduced in 1983, in order to allow the combination of the optical astrometry solution for universal time with the VLBI results which stated to be available. It was shown that the introduction of the IAU 1980 Theory of Nutation had a negligible effect on the pole coordinates, but corrected a quasi-annual error of maximum amplitude $1.8 \mathrm{~ms}$ on universal time (Capitaine and Feissel, 1983).

The other series have been regularly reported to the BIH by the U.S. Defense Mapping Agency (Loppler), the Smithsonian Astrophysical Observatory (SLR), the University of Texas Center for Space Research (SLR), the U.S. Naval Observatory (CERI), and the U.S. National Geodetic Survey (VLBI). Series covering several years have also been provided by some of these institutes, and by the Centre d'Etudes et de Recherches Geodynamiques et Astronomiques (LLR), The Massachusetts institute of Technology (LLR), the Jet Propulsion Laboratory (LLR and VLBI).

Prior to the combination, the error spectra of the individual time series are determined, under the view-point of both the deterministic and the random errors. Only the optical astrometry and the CERI time series require a seasonal correction (annual and semiannual terms). All series are brought to the BIH System by the correction of a bias (Feissel, 1982). The data of the individual series are weighted according to an unbiased estimate of their uncertainty, derived from the internal analysis of each of the series.

Over the time interval 1982-1984, the contribution of the data from the space techniques has been drastically increased. F or polar motion, SLR has contributed over 50\% of the total weight. VLBI has had a continuously increasing contribution, in particular for universal time, where it provides about $90 \%$ of the total weight in 1984 . The precision of the final combined solution at five-day interval for 1984 is estimated to 0.0005 s for universal time and $0.002^{\prime \prime}$ for the pole coordinates.

The final combined solution is computed for its publication in the Annual Report, issued in June of the year following the year covered. However, when the accumulation of new or improved results justifies it, a revised version of this combination is performed. This was the revised series obtained for optical astrometry was combined from 1978 onwards with the space geodesy series then available. Figure 1 shows the evolution of weights of the different techniques in the final solution at five-day interval.

Besides this final combined solution, the BIH has continued the implementation and the dissemination of two operational solution, available with a shorter time delay: the weekly Rapid Service and the monthly Circular D. computed with the same algorithm as the final solution, applied to the data available to the BIH in due time (4-10 days for the Rapid Service, 4-8 weeks for Circular D). Table I gives the quadratic mean of the differences of these solutions with the standard smoothings of the final solution, for 1983.

Table 1 - Agreement of the operational solutions with the final one (standard smoothing)

\begin{tabular}{l|lll} 
Solution Parameter & UTI & $X$ & $Y$ \\
\hline Rapid Service & $0.0019 \mathrm{~s}$ & $0.010^{\prime \prime}$ & $0.008^{\prime \prime}$ \\
Circular D & $0.0009 \mathrm{~s}$ & $0.004^{\prime \prime}$ & $0.003^{\prime \prime}$
\end{tabular}




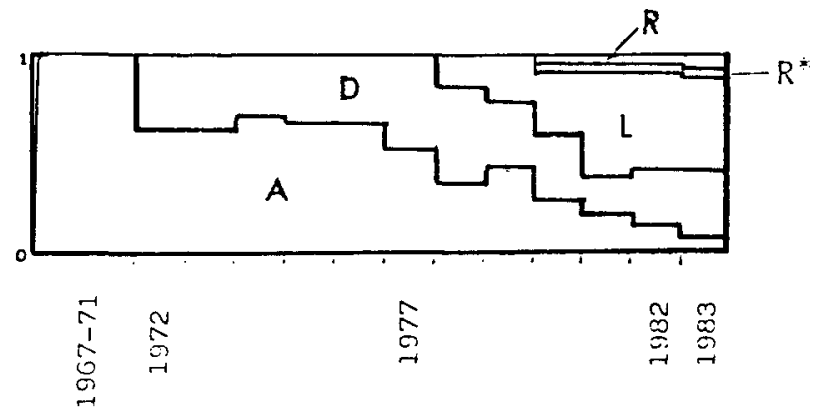

UNIVERSAL TIME

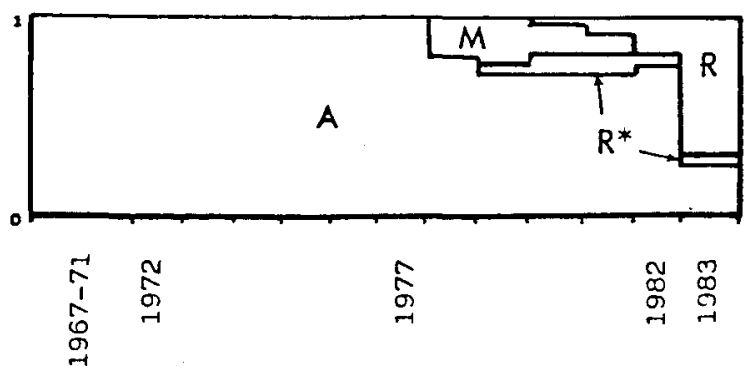

Figure 1. BIH combined solution for the Earth's rotation; relative weights of the techniques.
A Optical astrometry
D Doppler tracking of satellites
L Satellite laser ranging
$M$ Lunar laser ranging
R VLBI
$R^{*}$ Connected Radio Interferometry
This figure refers to Table $6(6 \mathrm{C})$ of the Annual Report of the BIH. 
Analyses.

The stability of the BIH Earth Rotation results since 1962 has been evaluated by internal analysis and by comparison with space geodesy results obtained independently. The BIH series show no detectable drift, the upper limits found being 0,0001 s/y for universal time and 0.0003 " for the coordinates of the pole.

The random error spectra of the independent series of the ERP have been investigated, for sampling times ranging from five days to one year (Feisse., 1984). It was shown that the hierarchy of stabilities of the different series is not identical in the short term and in the long term. This confirms the validity of the choice made earlier by the BIH to use different systems of weighting for the long term and the short term stability of their results (Guinot and Feissel, 1968).

A new solution by optical astrometry has been computed for the years 1962-1981, on the basis of revised series of time and latitude sent to the BIH by numerous stations. This work was accomplished by and astronomer of Shanghai Observatory, in stay at the BIH in 1982-1984 ( $\mathrm{Li}, 1984)$. The complete observational data consisted in 500000 determinations of time and latitude, organized in 198 time series obtained by 136 stations. The ERP were obtained at five-day interval in a global adjustment, together with the classical auxiliary unknowns $z$ and $w$ with 2600 "group unknowns" to free the solution from catalog or local errors. The solution is published in the Annual Report for 1984.

Experimental determinations of the ERP from SLR observations have been obtained, in cooperation with the Groupe de Recherches de Geodesie Spatiale (GRGS). The results are published in the MERIT reports. The construction of normal points to condense to raw observational data has been studied (Gambis, 1982, 1984a).

Several analyses of the BIH results have been performed;

- The amplitudes of the principal and fortnightly terms of nutation have been obtained with a 0"U0l percision using $z$ and $w$ data from 1962.0 to 1981.0. They are in good agreement with the 1980 IAU theory of nutation except the principal term in obliquity which shows a difference of 0"007 (Capitaine and Xiao, 1982).

- A time-varying anomaly of the response of the Earth to the Mr zonal wave has been detected in the UTl results (Capitaine et al. 1984) which could be of oceanic or atmospheric origin.

- The high correlation of the short term and medium term (30 days to 2 years) variations of the duration of the day with the atmospheric angular momentum has been established (Feissel, Rosen et al., 1982; Nitschelm, 1983).

- It has been shown that short term oscillations of the duration of the day are always present, with total amplitudes of the order of $1 \mathrm{~ms}$ and recurrence times between 20 and 60 days (Feissel and Nitschelm, 1984).

Theoretical aspects of the rotation of a deformable Earth with a fluid core have been considered, leading to an explicit formulation of the nutation in terms of the Earth model and of the considered axis (Capitane, 1982).

The use of Guinot's non rotating origin was investigated, in particular with respect to the definition of UTl (Aoki et al, 1982). It has been pointed out that the definition of the Celestial Ephemeris Pole (newly adopted by IAU) needs a clarification concerning the strictly diurnal term (Capitaine et al, 1984).

A collective program of time and latitude stars was proposed for observation by the ESA satellite HIPPARCOS (Feissel, Aoki et al., 1982). It includes 6082 stars coming from 39 lists provided by 24 instutes. The program was accepted by ESA with some minor 
restrictions.

In cooperation with the French Institut Geographique National (IGNF), the relationships between the terrestrial frames to which the different series of ERP obtained by space geodesy are referred were studied. As a result of this study, it was proposed that the BIH terrestrial system be redefined on the basis of the coordinates of the sites where space geodesy stations are operated in programs for the determination of the Earth's rotation (Boucher and Feissel, 1984).

\section{MERIT Campaign.}

The $B I H$ acted as the coordinator of MERIT project, in particular during the main observing campaign, from 1983 September 1 to 1984 October 31. The different tasks accomplished in this framework were as follows.

- Edition of the final results of the short campaign, (August-October 1980), included in the Report on the short campaign (Wilkins and Feissel (eds), 1982).

- Collection of the precise description and geodetic ties of the sites involved in the measurement of the Earth's rotaion (Feissel and Wilson (eds), of MERIT Sites (DOMES) is kept up-to-date.

- Collection of the Sets of Station Coordinates (SSC) defining the terrestrial frame for each series of EKP.

- Collection of the series of ERP obtained during the campaign by the Operational Centres (see Table 2) and of the series obtained after the end of the campaign by the Analysis Centres.

- Maintainance of a data bank for storing the collected data, dissemination of copies on request.

- Issuing of results from, and information on, the campaign, in the MERIT Monthly Circular, distributed to 180 addresses.

\section{Report of the International Polar Motion Service}

During the main campaign in Project MERIT, the IPMS acted as an analysis, as well as an operational center of optical astrometry.

Prior to the commencement of the main campaign of Project MERIT, the IPMS, as an analysis center of optical astrometry, developed a new method for estimating an optimumly smoothed series of Earth orientation parameters (EOP's: polar motion, UTI and l.o.d.) with one-day interval. This method is based on a firm statistical basis which uses Akaike's information criterion for selecting optimum smoothing parameters (Manabe et al. 1982; Yokoyama 1984a).

Nominal rms errors of the IPMS daily EOP's were estimated as $+/-0.003$ " for polar motion and $+/-0.2 \mathrm{~ms}$ for both UTl and l.o.d. Due to the improvement of the analysis technique, precisions of the EUP's derived from optical astrometry were remarkably improved.

This method is still being improved to determine variations of the station coordinates simultaneously with EOP's. The improved method is expected to bring out maximum possibility of optical astrometry as a technique for monitoring Earth rotation.

The IPMS daily EOP's have been published in the succeeding issues of the Monthly Note of the IPMS since 1983 No. 9, in place of the monthly mean EOP's which had so far 
been published. The new series of the daily EOP's have been reproduced in the Monthly Circular of Project MERIT published by the BIH and also in the Earth Orientation Bulletin published by the USNO.

Table 2 - MERIT Campaign (1983 Sep 1 - 1984 Oct 31)

Results received by the Cooordinating Centre

\begin{tabular}{|c|c|c|c|c|}
\hline Earth Rotation & Parameters & Interval & $\begin{array}{l}\text { First } \\
\text { Transmission }\end{array}$ & Terrestrial Frame \\
\hline ERP(NGS) $83 R Q L$ & $x, y$, UTl & $5 d$ & Nov $83(1)$ & SSC(NGS) $83 \mathrm{R} 01$ \\
\hline ERP(NGS) $83 \mathrm{R} \mathrm{QL}$ & $x, y$, UT 1 & $5 d$ & Nov $83(1)$ & SSC(NGS) $83 R 01$ \\
\hline EKP(NGS) $83 \mathrm{~K} \mathrm{QL}$ & $x, y, \cup T 1$ & $5 d$ & Jul 84 (1) & \\
\hline $\operatorname{ERP(JPL)} 82 \mathrm{R} 01$ & , UTO & $5-7 d$ & Mar 84 & $\mathrm{SSC}(\mathrm{JPL}) 82 \mathrm{R} 05$ \\
\hline Green Bank Interf. & , UTO & $3 d$ & Sep 83 (1) & \\
\hline Green Bank Interf. & UTO & $3 d$ & Apr $84(1)$ & \\
\hline ERP(CEKGA)83 M 01 & UTO & $1-33 d$ & May 84 & SSC(CERGA) $83 \mathrm{M} 01$ \\
\hline ERP(CSR) $81 \mathrm{~L} \mathrm{Ol}$ & $x, y, D$ & $5 d$ & Sep $83(1)$ & SSC(CSR) 81 L 01 \\
\hline ERP(CSR) 84 L 02 & $x, y, 0$ & $5 d$ & May $84(1)$ & \\
\hline ERP(GRGS) $84 \mathrm{~L} 01$ & $x, y, 0$ & $5 d$ & Jun 84 & SSC(GRGS) $84 \mathrm{~L} 01$ \\
\hline EKP(DNA) $77 \mathrm{D} 01$ & $x, y$, & $2 d$ & Sep $83(1)$ & \\
\hline ERP(DMA) 79 D 01 & $x, y$, & $2 d$ & Sep $83(1)$ & \\
\hline EKP(DMA) $82 \mathrm{D} 01$ & $x, y$, & $2 d$ & Sep $83(1)$ & \\
\hline$E K P(B I H) \quad 83$ A 02 & $x, y$, UTl & $5 d$ & Sep $83(1)$ & $\operatorname{SSC}(B I H) 83 \mathrm{~A} 01$ \\
\hline$E K P(B I H) 84$ A 02 & $x, y, \cup T 1$ & $5 d$ & Feb $84(1)$ & $\operatorname{SSC}(B I H) 84 A 01$ \\
\hline ERP(IPMS) $83 \mathrm{~A}$ & $x, y, \cup T 1, D$ & Id & Nov 83 & \\
\hline
\end{tabular}

(1) Results in the current combined ERP solution of the BIH. UTC,

The Monthly Note of the IPMS also gives daily mean values of latitude and UTl-

The IMIPS daily EOP's from 1962 were computed based on the system of MERIT Standards. These are in preparation for print. Magnetic tape versions of the results were already distributed to several institutions on request.

Intercomparison of the IPNIS daily EOP's was made with those estimated by other techniques and centers, as well as by atmospheric data (Yokoyama 1984; Hara and Yokoyama 1984). It turned out that the UTl and l.o.d. determined from optical astrometry agree far better than has been improperly believed. On the other hand, polar motion results show systematic errors of a few decimeters from the VLBI and SLR results, probably due to large observational errors of individual instruments.

Uaily values of the l.o.d. caused by the zonal wind and redistribution of atmospheric mass have been computed since September 1983 with a month delay using the global analysis data compiled at the Japan Meteorological Agency (Naito and Yokoyama 1984). The results are in good agreement with those computed at the National Meteorological Agency of the USA. This series of the l.o.d. will soon be dissiminated regularly. 
In accordance with the official adoption of the IAU 1976 System of Astronomical Constants and IAU 1980 Theory of Nutation from January 1, 1984 onwards, the IPMS, in collaboration with the Tokyo Astronomical Observatory and the Japan Hydrographic Department, developed algorithms and computer softwares to calculate apparent places of stars for common use in the reduction of observations by optical astrometry at the individual stations (Yokoyama 1983; Yokoyama 1984b). Magnetic tape versions of the computer softwares were distributed to every country where optical astrometry instruments are in operation. Thus, apparent places of stars are being computed at individual stations based on the same theoretical basis.

In cooperation with the USNO, all PZT observations of Washington and Mizusawa were re-reduced using the system of MERIT Standards and the unified catalog of star positions and proper motions was compiled in the system of $F K 4$. This work was being extendedly applied to PZT observations of other stations.

In collaboration with the Tokyo Astronomical Observatory, improvement of the computer software for satellite laser ranging, which was originally developed at the SAO using an analytical method, is in progress. Aimed goal is to achieve the precision comparable to that already achieved by other softwares using the method of numerical integration.

\section{IAU/IUGG Joint Working Group on the Rotation of the Earth (MERIT)}

The IAU/IUGG Joint Working Group on the Rotation of the Earth has been very active since the IAU General Assembly in Patras in organizing the MERIT campaigns of observation and analysis. It has cooperated closely with the IAU/IUGG Joint Working Group on the Establishment and Maintenance of the Conventional Terrestrial Reference System (CUTES). The Second MERIT Workshop was held at the Royal Greenwich Observatory on 1983 May 15 - 19 to review the operational arrangements for the MERIT Main Campaign, which was held from 1983 September 1 to 1984 October 31, and for the COTES Intensive Campaign, which was held from 1984 April 1 to 1984 June 30. In addition to the monitoring of the rotation of the Earth (universal time or length of day and polar motion) by six different techniques, special attempts were made to obtain simultaneous high-precision observations by two or more techniques at selected sites and to determine the position of all sites by the Doppler technique.

Reports on the operations during the period of the main campaign and on the subsequent analyses of the data are published in the MERIT Monthly Circular which is prepared and distributed to participating organizations by the Bureau International de l'Heure. It is clear from the results that have already been obtained that Project MERIT has been successful in stimulating the development and regular use of new high-precision techniques (especially satellite laser ranging and very long baseline radio interferometry) for monitoring the rotation of the Earth; it has also led to an improvement in the quality of the results obtained by the optical astrometry. The project has generated a high level of cooperation, as exemplified by the adoption of MERIT Standards for constants and numerical models for use in the analysis of observations. The improved precision of the earth-rotation parameters has also made possible detailed comparisons with the variations in the angular momentum of the atmosphere that are of great interest to meteorologists. The extension of the MERIT campaign to provide additional data for COTES has emphasized the importance of monitoring earth-rotation for geodetic purposes, and the dense data sets obtained during the intensive campaign are likely to be valuable in many other studies.

The principal disappointment has been that the lunar laser ranging observations have been much fewer than had been hoped, and so it has been decided to hold a second intensive campaign in 1985 May to July so that the potential of LLR observations for monitoring earth-rotation can be properly assessed and so that the relationships between the reference systems can be better determined. The third MERIT Workshop will be held at Columbus, Ohio, on 1985 July $29-30$ and will be followed immediately by a conference for the presentation of the preliminary results from the MERIT-COTES campaigns. The 
implications of the results for the international services will be discussed at the IAU General Assembly in Delhi. In the meantime the operational arrangements developed for the campaigns will be continued, although the level of observation will not be so high.

\section{IAU/IAU Joint Working Group On The Establishment And Maintenance Of a Conventional Terrestrial Reference System (COTES)}

The International Association of Geodesy, IUGG/International Astronomical Union Joint Working group on the Establishment and Maintenance of a Conventional Terrestrial Reference System (CUTES) was established in 1981 following a resolution at the IAU Colloquium $\# 56$ on Reference Coordinate Systems for Earth Dynamics, where it was recommended:

that a working group be established by the Presidents of IAU Commissions 4, 19 and 31 and the President of the IAG to prepare a proposal for the establishment and maintenance of a conventional terrestrial reference system.

The membership of COTES is as follows: E. M. Gaposchkin (USA), B. Guinot (France), B. Kolaczek (Poland), J. Kovalevsky (France), D. D. McCarthy (USA), W. G. Melbourne (USA), P. Melchoir (Belgium), K. Yokoyama (Japan), Y. S. Yatskiv (USSR), I. I. Mueller (USA), Chairman.

In 1981/82 CUTES prepared a proposal entitled "Reference Frame Requirements and the MERIT Campaign" for the purpose of planning the observations during Project MERIT in such a way that they contribute everything possible to studies leading to the establishment and maintenance of the new conventional terrestrial reference system to be proposed. In particular, the necessity to identify precisely the coordinate system being used implicitly by each of the MIERIT networks was noted. It would then be necessary to establish relationships between these systems.

The proposal in a somewhat modified form was carried out through the Main Campaign of Project MERIT between September, 1983, and October, 1984, especially during an intensive campaign in the Spring of 1984, carried out especially for the purposes of CUTES. The results of the observations are being analyzed and are to be reported on at the MIERIT Workshop in July, 1985, to be held at The Ohio State University in Columbus, Ohio, where a proposed resolution will be prepared if at all possible for adoption at the IAU General Assembly in India in November, 1985, and subsequently at the next General Assembly of the IUGG.

\section{IAG/COSPAR International Radio Interferometric Surveying (IRIS) Sub-Commission}

The International Radio Interferometric Surveying (IRIS) joint sub-commission of the International Association of Geodesy (IAG) and the Committee on Space Geodesy (COSPAR) implemented an Earth rotation monitoring service, based on Very Long Baseline Interferometry (VLBI). Since January 1984 the IRIS observations have been made regularly at five-days intervals, using three-stations in the United States (Westford, Massachusetts; George R. Agassiz Station, Texas; and Richmond, Florida) and the Wettzell Observatory, in Bavaria, Federal Republic of Germany (FRG). The Onsala Space Observatory, Sweden, participates in one IRIS session each month. The observations are correlated at the Haystack, Massachusetts or Max Planck, FRG, correlators, and the final reductions and analysis to derive the $x$ and $y$ components or polar motion and UTI are performed at the National Geodestic Survey, Rockville, Maryland. The polar motion and UTl time series are distributed monthly through the Bureau International de l'Heure and by direct mailing in the form of the IRIS Bulletin A. The Formal Standard errors of the time series are typically 1 to 2 milliseconds of arc in $x$ and $y$, and 0.05 to 0.1 milliseconds in UTl. Experiments with the Westford-Wettzell interferometer have indicated that it is feasible to monitor UTl to 
the U.1 millisecond level on an hourly, or even shorter, basis. A 90-day series of such measurements were made in support of the MERIT intensive campaign, during April - June, 1984.

\section{REFERENCES}

Bernacca P. L. (Ed.), 1983 : Processing of Scientific Data from the E. S. A. Astrometry Satellite HIPPARCOS. F.A.S.T. held in Asiago, Italy.

Bondareva M. B., Yurkina M. I., 1984 : Geodesiya i Kartografiya, 5, p.9-13 (in Russian). Boucher C., Feissel M., 1984 : Paper presented to International Symposium on Space

Feissel, M., \& P. Wilson (eds), 1983. MERIT Campaign: Connection of reference frames: Implementation Plan. Paris: Bureau International de l'Heure

Kolaczek B., Brzesinski A., Kosek W., King R. W., 1984 : Paper presented to International Symposium on Space Techniques for Geodynamics, Sopron, Hungary. Techniques for Geodynamics, Sopron, Hungary.

Lehmann, M., 1982: The Program of Observations with the Danjon Astrolabe at Astromonical Latitude Observatory in Borowiec, Mitteilung des Lohrmann, Observatoriums der Technischen Universitat Dresden.

Melboume, W. (ch), 1983. Project MERIT Standards. U. S. Naval Observatory Circular No. 167.

Molodenskij S. M., 1984 : Prilivy, nutatsiya i vnutrenneye stroeniye Zemli. Moskwa, 213 pp. (in Russian).

Parijskij N.N., 1985 : In: Povtornye gravimetriche nablyudeniya. Moskwa (in Russian).

Wilkins, G. A. (ed.), 1984. Report on the Second MERIT Workshop and on other activities in 1983 Herstmonceux, UK: Royal Greenwich Observatory.

Yokoyama, K. 1983, Report of Second MERIT Workshop, Greenwich, GBR, May.

Yokoyama, K. 1984, Guidelines for the Reduction of Optical Astrometry Observations during the Main Campaign in Project MERIT. Publ. Int. Latit. Obs. Mizusawa, 17, 2. 